\title{
Über Versuche am Elektronenzyklotron
}

\author{
Von Helmut Salow ${ }^{1}$ \\ (7. Naturforschg. 2a, 389-395 [1947]; eingegangen am 26. November 1946)
}

\begin{abstract}
Es wird eine Apparatur beschrieben, die dem Zyklotron nachgebildet, aber geeignet ist, Elektronen zu beschleunigen. Es werden die Elektronenbahnen, die sich in- und außerhalb des magnetischen Resonanzfeldes ergeben, sichtbar gemacht und photographiert. Ihre Form und Intensitätsverteilung wird mit einem Auffänger gemessen. Aus der Zahl und Lage der Elektronenbahnen wie aus ihrem Intensitätsabfall lassen sich Schlüsse auf die Fokussierungseffekte im Elektronenzyklotron ziehen bei Berücksichtigung der Phasenlage zwischen Magnet- und HF-Feld.

Zum Schluß werden Angaben gemacht über die Abhängigkeit der Fokussierungseigenschaften des $H F$-Feldes vom Eintrittswinkel der Elektronen in den Halbdosenraum. Es zeigt sich, daß bei richtiger Wahl des Eintrittswinkels annähernd alle Elektronen, die sich aus der Kathode herausziehen lassen, im Elektronenzyklotron fokussiert und beschleunigt werden können.
\end{abstract}

$\mathrm{D}$ ie Theorie des Zyklotrons, seine Arbeitsweise und seine Fokussierungsbedingungen sind oft der Gegenstand eingehender Untersuchung gewesen $^{2-6}$, so daß von theoretischer Seite die Arbeitsvorgänge im Zyklotron als geklärt angesehen werden können. Nun hat D ä n z e r ${ }^{7}$ in einer Arbeit nachgewiesen, daß das Zyklotronprinzip sich in gleicher Weise sowohl auf schwere Teilchen, wie Deuteron- und Heliumionen, als auch auf Elektronen anwenden läßt. Freilich lassen sich mit Elektronen, solange das Magnetfeld zeitlich konstant bleibt, keine hohen Energien gewinnen, da schon bei Spannungen von mehreren 1000 Volt so hohe Prozentsätze der Ruhenergie des Elektrons erreicht werden, daß die Resonanz- und die Fokussierungsbedingung nicht mehr gleichzeitig erfüllt werden können. Es lassen sich aber viele Fragen, die einer theoretischen Behandlung nur schwer oder gar nicht zugänglich sind, am Elektronenzyklotron in experimentell einfacher Weise studieren. Das Ziel der vorliegenden Arbeit ist es daher, über einige Punkte, wie Form und Intensitätsverteilung der ersten Bahnen, ihr Verhalten in und außerhalb der Resonanz, ihre Abhängigkeit von den Anfangsbedingungen, worüber bisher nicht viel bekannt geworden ist, durch das Experiment am Elektronenzyklotron etwas näheren Aufschluß zu erhalten.

1 Z. Zt. (24) Bargteheide, Holstein. Die Arbeit wurde in der For schungsanstalt der Deut $\mathrm{schen} \mathrm{Reichspost} \mathrm{in} \mathrm{Miersdorf} \mathrm{bei} \mathrm{Berlin} \mathrm{ge-}$ macht und war von der Redaktion der Physikalischen Zeitschrift zur Veröffentlichung angenommen worden. Sie konnte aber nicht mehr im Druck erscheinen.

\section{Die A p paratur}

Abb. 1 zeigt einen Schnitt durch die Versuchsanordnung. Innerhalb zweier großer Helmholtz-Spulen $(B)$ befindet sich ein evakuierbarer Glaskolben $(C)$, in den durch 3 Glasschliffe die Halbdosen $(H)$ mit Le che r-System $(E)$, die Kathode $(G)$ und der Auffänger $(J)$ eingeführt sind. Da im wesentlichen die ersten Elektronenbahnen untersucht werden sollten, bei denen eine magnetische Fokussierung noch keine Rolle spielt, wurde von vornherein ein möglichst homogenes Magnetfeld angestrebt, das durch zwei Helmholtz-Spulen mit einem inneren Radius von $200 \mathrm{~mm}$ realisiert wurde. In der Mittelebene der Spulen, am Ort der Elektronenbahnen, war das Feld im Bereich eines Radius von $60 \mathrm{~mm}$ völlig homogen. Bei einem Radius von $90 \mathrm{~mm}$ fiel das Feld um $2 \%$ ab. Zur Erzeugung der Hochfrequenz-Spannung (HF-Spànung) wurde ein kleiner 20-Watt-Sender benutzt, der bei einer Wellenlänge von $6,3 \mathrm{~m}$ arbeitete( das zugehörige Magnet-Resonanzfeld beträgt dann 16,9 Gauß) und mit dem an den Halbdosen bis zu 300 Volt Spannung erzielt werden konnte. Das Lecher-System bestand aus Messingstäben von $7 \mathrm{~mm}$ Durchmesser. Mit dem Kurzschlußbügel $(F)$ war das System leicht in Resonanz zu bringen und die Spannung zu symmetrisieren, die ständig mit einem $H F$-Voltmeter kontrolliert wurde. Um eine gute Beobachtung der Elektronenbahnen zu ermöglichen, waren die Halbdosen aus $2 \mathrm{~mm}$ weitem Bronzenetz gearbeitet. Sie hatten einen Radius von $90 \mathrm{~mm}$, eine lichte Höhe von $40 \mathrm{~mm}$. Ihr Abstand voneinander betrug $20 \mathrm{~mm}$. Zwischen den Halbdosen war die Kathode beweglich angeordnet. Sie bestand stets aus einem mit Emissionspaste belegten

2 M. E. Rose, Physic. Rev. 53, 392 [1938].

3 R. R. Wils on, Physic. Rev. 53, 408 [1938].

${ }^{4}$ R. R. Wils on, J. appl. Physics 11, 781 [1940].

5 L. H. Thomas, Physic. Rev. 54, 580, 588 [1938].

${ }_{6}$ W. W i e be, Z. Physik 122, 451 [1944]. .

‘ H. Dänzer, Ann. Physik 41, 485 [1942]. 
Platindraht oder Platinblech. Ihre Form und Anordnung mit zusätzlichen Blenden wurde vielfach verändert und dem vorliegenden Zweck angepaßt. Am besten bewährte sich eine Anordnung, bei der die Kathode direkt vor eine Blende gestellt wurde, die mit einer Halbdose verbunden war und in der Mitte einen Schlitz trug, durch den die Elektronen in den Halbdosenraum gesaugt wurden.

Der bewegliche Auffänger $(J)$ gestattete die Elektronendichte in den einzelnen Bahnen auszumessen. Es wurde ein Metall-Auffangkäfig benutzt, dessen innere metallische Belegung den eigentlichen Auffänger darstellte, während die äußere, durch Glimmer isolierte liche Phasenbreite beim Start der Elektronen müssen gering sein, wenn die Bahnen bis zu mehreren Umläufen verfolgt werden sollen. Die räumliche Begrenzung der Anfangsphase kann leicht durch ein Blendensystem vor einer Halbdose bewirkt werden. Die zeitliche Begrenzung wird durch das Zusammenwirken des HF-Feldes und der Charakteristik der Kathode von selbst erreicht. Eine Beeinflussung des Elektronenstrahles durch positive Raumladung nach Art eines Fadenstrahles tritt bei einem Gasdruck von $10^{-4} \mathrm{~mm} \mathrm{Hg}$ noch

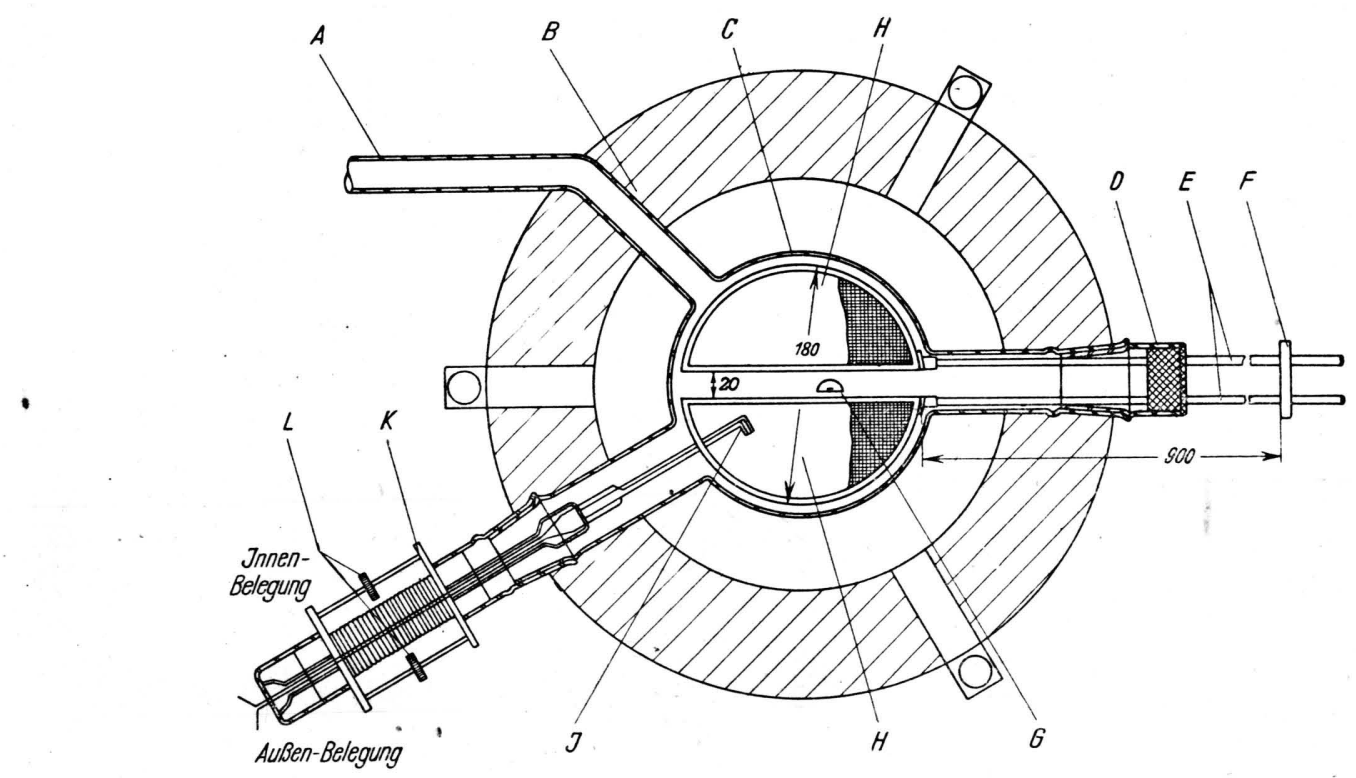

Abb. 1. Schnitt durch die Apparatur.

Belegung geerdet war. Wenn die Elektronenbahnen sichtbar gemacht werden sollten, wurde ein Gasrest von etwa $10^{-4} \mathrm{~mm} \mathrm{Hg}$ in der Apparatur belassen. Höhere Gasdrucke konnten nicht angewendet werden, weil dann eine Gasentladung zwischen den Elektroden entstand, die die HF-Spannung sofort zusammenbrechen ließ. Die photographischen Aufnahmen wurden zum Teil yon vorne, zum Teil über einen Spiegel von unten her gewonnen. Wegen der geringen Leuchtdichte der Erscheinung mußten die Aufnahmen im allgemeinen eine halbe Stunde belichtet werden.

\section{Die Elektronenbahnen in Resonanz}

Sollen die Bahnen im Elektronenzyklotron dem Auge sichtbar gemacht werden, so müssen mehrere Voraussetzungen erfüllt sein. Damit nur ein Spiralsystem entsteht und die Übersichtlichkeit erhalten bleibt, darf die Kathode nur nach einer Seite emittieren. Die räumliche Divergenz des Elektronenstrahles im ersten Umlauf und die zeit- nicht auf. Alle Fokussierungseffekte sind durch die elektrische Linse im Halbdosenspalt hervorgerufen. Unter solchen Bedingungen sind die Bilder I, II und IV der Abb. 2 (s. Tafel, S. 388 b) bei verschiedenen $H F$-Feldern entstanden. Der helle Punkt in der Mitte der Aufnahme bezeichnet den Ort der Kathode, die dunklen Balken quer über dem Bild die Ränder der Halbdosen, deren Netzstruktur gerade noch angedeutet wird.

Das Spiralsystem der Elektronenbahnen läßt sich sehr gut durch ein Aneinanderfügen von Halbkreisen mit wachsendem Radius beschreiben, derart, daß die Mittelpunkte der Halbkreise auf einer Parallelen zu den Halbdosenkanten durch die Kathode liegen. Abb. 3 gibt ein Bild der Wanderung der Halbkreismittelpunkte auf dieser Parallelen. Die Bahn beginnt in $K_{1}$, dem Ort der Kathode. Die ungeraden Ziffern bezeichnen die Bahnmittelpunkte der unteren Halbebene, die ge- 
raden Ziffern die der oberen Halbebene. Die Differenzen $\Delta r$ der Bahnradien $(\Delta r$ ist der Abstand zweier aufeinander folgender Mittelpunkte) nehmen etwa mit $1 / r$ ab, wie theoretisch zu erwarten, da der Energiezuwachs $\Delta V$ bei einem Spaltdurch-

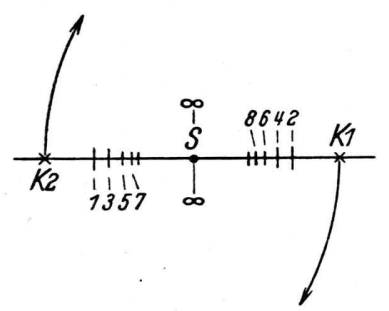

Abb. 3. Wanderung der Halbkreismittelpunkte mit der -Umlaufzahl. $K_{1}$ u. $K_{2}$ Ort der Kathoden. $S$ Symmetriepunkt des Spiralsystems der Bahnen.

tritt proportional zu $r \cdot \Delta r$ verläuft. Ein genauer Vergleich mit der Theorie wurde so durchgeführt, daß zunächst der Abstand der Bahnmaxima voneinander photometrisch aus den Aufnahmen ermittelt und dann mit dem entsprechenden, theoretisch zu erwartenden Energiezuwachs verglichen wurde. Letzterer läßt sich leicht durch graphische Integration aus dem bekannten Feldverlauf auch für solche Bahnen errechnen, in denen die Resonanzbedingung nicht streng erfüllt ist. In allen Fällen wurde eine befriedigende Übereinstimmung zwischen Experiment und Rechnung gefunden. Für unendlich großen Radius würden sich die Mittelpunkte im Symmetriepunkt $S$ des Spiralsystems treffen. Wenn die äußeren Bahnen im Zyklotron rotationssymmetrisch zum Magnetfeld liegen sollen, muß der Symmetriepunkt des Spiralsystems mit dem Mittelpunkt des Magnetfeldes zusammenfallen. Die Stellung der Kathode liegt also außerhalb der Feldmitte. Ihr Abstand davon hängt vom ersten Bahnradius und damit von der Spannung an den Halbdosen ab. Will man im Zyklotron beide Halbperioden zur Beschleunigung von Teilchen ausnutzen, so muß eine zweite Kathode an der Stelle $K_{2}$ eingeführt werden, die den gleichen Abstand vom Symmetriepunkt $S$ hat wie $K_{1}$. Das Symmetriezentrum und die Halbkreismittelpunkte blęiben erhalten, nur vertauschen die geraden und ungeraden Ziffern gerade ihren Platz.

In allen Aufnahmen fällt der scharfe äußere Rand der Bahn besonders bei den ersten Umläufen auf, während die Intensität nach innen wesentlich langsamer abfällt. Der äußere Rand der Bahn, also der größte Krümmungsradius, entspricht der
Maximalenergie, die die Elektronen je Umlauf aufnehmen können, also den Elektronen, die mit der Phase $\Theta_{0}=0$ starten (vergl. Abb. 6). Alle anderen Elektronen, die früher oder später starten, also negative oder positive Phase besitzen, gewinnen geringere Energien und damit kleinere Krümmungsradien. Alle Bahnen zeigen nach den äußeren Umläufen zu einen starken Intensitätsabfall. Dieser Intensitätsabfall wird auf den Aufnahmen allerdings nur durch die geringere Anregungswahrscheinlichkeit der Gasmoleküle vorgetäuscht, die die Elektronen mit wachsender Energie erhalten. Den wahren Intensitätsabfall kann man aber leicht mit einer Sonde ausmessen, die sich vom Rande her senkrecht durch die Bahnen zum Symmetriepunkt verschieben läßt. Abb. 4 zeigt den auf diese Art gewonnenen Intensitätsverlauf bei $75 \mathrm{~V} H F$-Spannung an den Halbdosen. Die Abbildung enthält als ausgezogene Kurve den Auffängerstrom in Abhängigkeit vom Symmetriepunktabstand, als gestrichelte Kurve die daraus

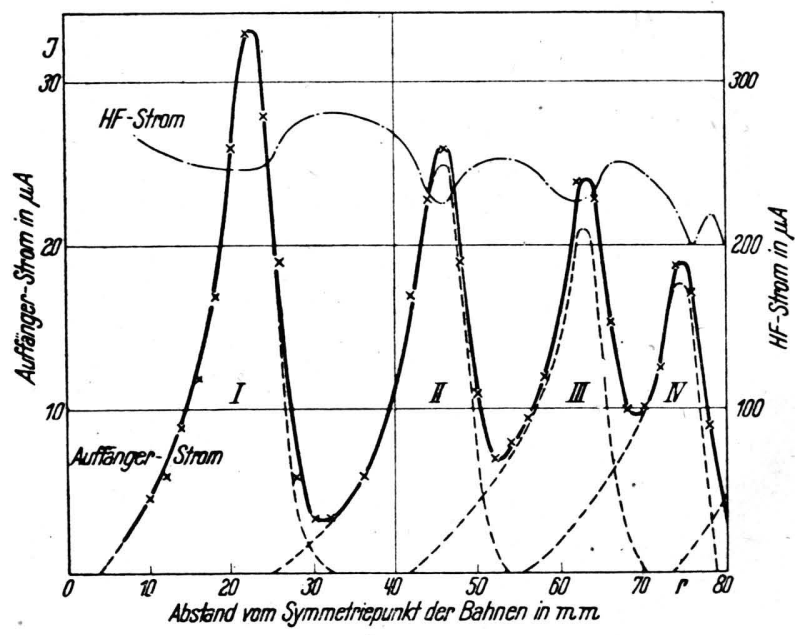

Abb. 4. Intensitätsquerschnitt durch die Bahnen bei $75 \mathrm{~V}$ HF-Spannung. Die gestrichelten Kurven geben die Intensitätsaufteilung auf die einzelnen Bahnen. Die strichpunktierte Kurve zeigt den von den Halbdosen aufgenommenen $H F$-Strom.

abgeleitete Aufteilung der Intensität auf die einzelnen Bahnen, als strichpunktierte Linie den zugehörigen $H F$-Strom auf die Halbdosen (an den Halbdosen gemessen), in dem sich die Maxima des Auffängerstromes als Minima abzeichnen. Der steile Abfall der Bahnen zu großen und der flache Abfall zu kleineren Radien tritt deutlich hervor. Man darf annehmen, daß die Breite und Form 
eines Bahnmaximums im wesentlichen von der Intensitätsverteilung in der zeitlichen Anfangsphase $\Theta_{0}$ bestimmt sind, da die räumliche Divergenz des Elektronenstrahles bei dem verwendeten Kathodensystem sehr klein wird und nur etwa $6^{\circ}$ beträgt.

Daß die Zahl der Elektronen, die in den einzelnen Bahnen kreisen, nach außen abnimmt, ist aus Abb. 3 ohne weiteres ersichtlich. Einen genaueren Aufschluß hierüber gewinnt man, wenn man die Intensitätsverteilung auch senkrecht zur Bahnebene mit dem Auffänger ausmißt. Das Maximum der Elektronendichte Iiegt natürlich in der Mittelebene der Bahn. Doch ist auch der Raum in der Nähe der oberen und unteren Kante der Halbdosen noch mit Elektronen erfüllt, wie man gut in Bild VII der Abb. 2 beobachten kann. Nach dem Ausmessen der Bahn in zwei Koordinatenrichtungen läßt sich durch mehrfache graphische Integration der gesamte Elektronenstrom bestimmen, der in einer Bahn kreist. So ergibt sich, daß im 1. Bahnmaximum $182 \mu \mathrm{A}$, im 2. $158 \mu \mathrm{A}$, im 3 . $144 \mu \mathrm{A}$ und im 4. $108 \mu \mathrm{A}$ fließen. Hier findet man also eine Abnahme der Elektronenzahl von der ersten bis zur letzten Bahn um nahezu die Hälfte. Dies Verhältnis ergibt sich auch bei anderen $\mathrm{HF}$ Spannungen an den Halbdosen, wenn die Elektronen senkrecht in den Halbdosenraum eintreten, so daß in diesem Intensitätsverlust ein grundsätzlicher Effekt gesehen werden muß. Er läßt sich am einfachsten durch das allmähliche Ausscheiden der Elektronen mit negativer Phase, die in der ersten Bahn noch enthalten sind, deuten, wie im Abschn. 4 noch eingehend erläutert wird.

\section{Die Elektronenbahnen außerhalb} der Resonanz

Alle bisher beschriebenen Versuche bezogen sich auf Elektronen, deren magnetische Umlauffrequenz sich in Resonanz mit dem $H F$-Feld befand. Verändert man jetzt das Magnetfeld bei fester Frequenz des $H F$-Feldes und mißt den Auffängerstrom, so entstehen die für das Zyklotron typischen Resonanzkurven. Sie hängen stark von der Größe der angelegten $H F$-Spannung ab. Die von, Dänzer ${ }^{7}$ angegebene Form der Resonanzkurven läßt sich beim vorliegenden Elektronenzyklotron nicht nachweisen, da das Magnetfeld auf der ganzen Bahn konstant ist und keine relativistische Massenänderung der Elektronen auftritt. Ebenso sollten nach Dänzer auch Elektronen mit sehr kleiner Anfangsenergie $(\sim 2 \mathrm{~V})$ in Resonanz den Auffänger noch erreichen können. Eine experimentelle Nachprüfung scheitert an der geringen Kathodenemission bei so niedrigen Spannungen. Abbildg. 5 zeigt die außerordentliche Schärfe der Resonanzkurve bei $20 \mathrm{~V}$ und ihre starke Verbreiterung bei Zunahme des $H F$-Feldes $(60 \mathrm{~V})$. Bei höherenFeldstärken machen sich die getrennten Bahnen mit mehreren Maxima in der Reso-

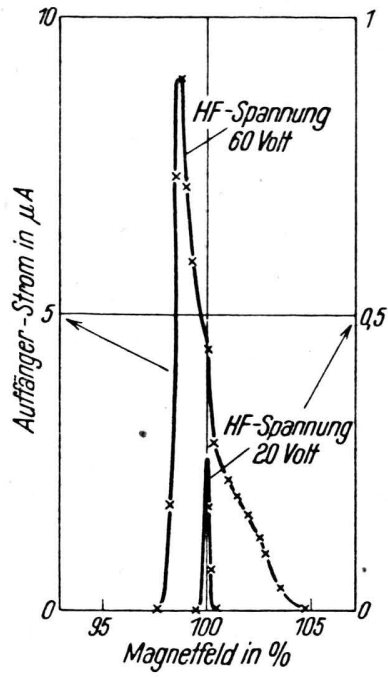

Abb. 5.

Resonanzkurven des Auffängerstromes bei Änderung des Magnetfeldes. nanzkurve bemerkbar.

Die Elektronenbahnen zeigen bei Magnetfeldern, die oberhalb oder unterhalb des Resonanzfeldes liegen, ein charakteristisches Verhalten. Als Beispiel hierfür sind die Bilder III bis VI der Abb. 2 reproduziert. Alle Elektronen, die außerhalb der Resonanz umlaufen, vergrößern ihre Phasendifferenz gegenüber dem Magnetfeld kontinuierlich bei jedem Umlauf. Sobald ihre Phasendifferenz $\pi / 2$ erreicht hat, werden sie nicht mehr beschleunigt, sondern treffen auf ein Gegenfeld, das den Bahnradius verkleinert und eine in den Ursprung zurücklaufende Spiralbahn erzeugt. In Bild III der Abb. 2 ist die Wiedergabe einer solchen rückläufigen Spiralbahn versucht worden. Diese Bahnen sind sehr schwer einzustellen und zu beobachten. Die Reproduktion besitzt deshalb nicht die Schärfe und Deutlichkeit der übrigen Aufnahmen. Immerhin sind zwischen der stärkeren normalen Spiralbahn Teilbahnen zu erkennen, die sich bei gleichem Umlaufssinn zu einem Spiralsystem zusammensetzen, das sich zur Kathode hin zusammenzieht. Solche rückläufigen Bahnen treten nur auf, wenn das Magnetfeld kleiner als das Resonanzfeld ist, da im anderen Falle ein Defokussierungseffekt die Zahl der Bahnen begrenzt.

Geht man zu stärkeren Abweichungen vom Resonanzfeld über, so erfüllen die Bahnen nicht 
mehr den ganzen Raum in den Halbdosen, sondern erreichen vorher einen maximalen Radius, den sie nicht mehr überschreiten können. Dieser Zustand ist in den Bildern IV, V und VI der Abb. 2 beschrieben. Alle Aufnahmen sind bei der gleichen $H F$-Spannung von $41 \mathrm{~V}$ gemacht. In Bild IV ist das Resonanzfeld $H_{r}$ eingestellt, in Bild V ein um 5,2\% größeres, in Bild VI ein um 7,7\% kleineres Magnetfeld als das Resonanzfeld. In Bild IV ist der ganze sichtbare Bereich mit 6 bis 7 Bahnen ausgefüllt, während in den Bildern V und VI nur noch 4 und 3 Bahnen erkennbar sind, die wegen des größeren oder kleineren Magnetfeldes einen entsprechend kleineren oder größeren Radius besitzen. Ebenso wird deutlich, daß die Bahnen für $H>H_{r}$ schärfer fokussiert werden und für $H<H_{r}$ ein verwascheneres Aussehen erhalten, als den Bahnen im Resonanzfall entspricht. Dies findet seinen Grund darin, daß für $H>H_{r}$ die Elektronen gleichsam vor dem $H F$ Felde herlaufen, also während des Durchtritts durch den Halbdosenspalt auf eine elektrische Linse stoßen, deren Spannung während des Durchlaufens zunimmt. Eine solche Linse wirkt aber für den Teil der Elektronen, die nicht genau in der Mittelebene laufen, wie eine Zerstreuungslinse. Diese werden daher nach und nach aus der Bahn abgelenkt und regen dabei das Restgas in der Umgebung der Bahnen, wie auf Bild V deutlich zu erkennen ist, zum Leuchten an. Im Gegensatz hierzu treffen die Elektronen bei einem Felde $H<H_{r}$ auf eine elektrische Sammellinse. Die Elektronen bleiben weitgehend in ihrer Bahn fokussiert und bilden einen dicken leuchtenden Ring, der die Kathode umschließt. Diese räumliche Erscheinung ist visuell eindrucksvoller $\mathrm{zu}$ beobachten, als sie durch die flächenhafte Photographie wiedergegeben werden kann.

4. Die räumliche und diezeitliche Anfangsphase des Elektronenstrahles

Die räumliche und die zeitliche Anfangsphasedas ist der Raumwinkel, unter dem die Elektronen die Kathode verlassen, und die Emissionszeit, in der die Elektronen von der Kathode emittiert werden - sind von entscheidender Bedeutung für die Ausbeute an fokussierbaren Elektronen und auch für den Verlauf der Elektronenbahnen. Aus der Charakteristik der Kathodenemission und aus dem zeitlichen Verlauf des HF-Feldes kann man ablesen, daß für eine Oxydkathode, die nach einer Halbdose emittiert, nur mit einer Emissionszeit gerechnet werden kann, die ein Viertel der ganzen $H F$-Periode beträgt.

Während die zeitliche Anfangsphase $\Theta_{0}$ durch die Kathode, das $\mathrm{HF}$-Feld und die Absauggeometrie unverändert festgelegt ist, läßt sich die räumliche Anfangsphase durch Drehung der Kathode um eine vertikale und eine horizontale Achse leicht ändern. Mit der räumlichen Anfangsphase sind im folgenden der Winkel $\&$ und der Winkel $\varphi$ gemeint, unter denen der Elektronenstrahl die Kathode verläßt. $\vartheta$ liegt in der Bahnebene und mißt den Winkel zwischen der $y$-Achse und der Anfangsrichtung des Strahles (vergl. Abb.6), $\varphi$ liegt

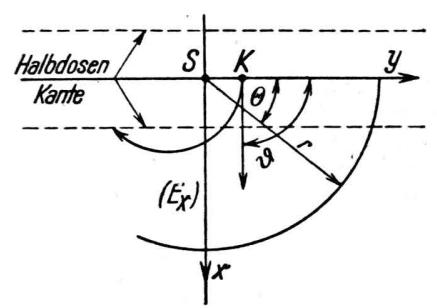

Abb. 6. Die Phase $\Theta$ eines Elektrons beim Umlauf und die räumliche Anfangsphase $\vartheta$ beim Start der Elektronen. $E_{x}$ elektrische Feldstärke in der $x$-Richtung. $K$ Ort der Kathode, $S$ Symmetriepunkt der Bahnspiralen.

in der Ebene senkrecht hierzu und bezeichnet die Abweichung des Strahles von der Mittelebene. Zunächst soll nur der Winkel $\vartheta$ in der Bahnebene betrachtet werden.

Man macht sich leicht klar, daß bezüglich der Phasenlage des Elektrons beim Spaltdurchtritt der Winkel $\vartheta$ und die zeitliche Anfangsphase $\Theta_{0}$ die gleiche Wirkung ausüben und somit vertauschbar sind, und zwar entsprechen in der hier verwendeten Schreibweise $\Theta_{0}=0^{\circ}$ dem Winkel $\vartheta=90^{\circ}$, so daß, falls die zeitliche Anfangsphase $\Theta_{0}$ von $-90^{\circ}$ bis $+90^{\circ}$ läuft, der äquivalente Einfallswinkel $\vartheta$ der Elektronen von $0^{\circ}$ bis $180^{\circ}$ läuft. Man kann also durch Änderung des Eintrittswinkels \& der Elektronen in die Halbdosen die zeitliche Anfangsphase $\Theta_{0}$ beliebig variieren. Damit hat man aber die Möglichkeit, den zeitlichen Emissionsbereich derart $\mathrm{zu}$ verschieben, daß die Elektronen in der Gesamtheit bei jedem Spaltdurchtritt auf eine Sammellinse oder auf eine Zerstreuungslinse treffen. Auf diese Art lassen sich die Fokussierungs-Eigenschaften des HF-Feldes für alle Elektronen, die an einem ganzen Viertel der vollen Periode die Kathode verlassen, aus- 
nutzen und durch das Elektronenzyklotron bringen. Es wurden in dieser Richtung Versuche durchgeführt, bei denen die Kathode und die Saugblende unter verschiedenen $\diamond$-Winkeln zur Halbdose eingestellt waren. Mit dem Auffänger wurde dann ein Intensitätsquerschnitt durch die ersten vier Elektronenbahnen bei $75 \mathrm{~V} H F$-Spannung wie in Abschn. 2 vorgenommen, aus dem die Elektronenströme, die auf die einzelnen Bahnen entfallen, wieder durch graphische Integration gewonnen werden konnten. Die Ergebnisse sind in Tab. 1 zusammengefaßt.

\begin{tabular}{|r|c|c|c|c|c|}
\hline$\vartheta$ & $\begin{array}{c}\text { I. } \\
\text { Bahn }\end{array}$ & $\begin{array}{c}\text { II. } \\
\text { Bahn }\end{array}$ & $\begin{array}{c}\text { III. } \\
\text { Bahn }\end{array}$ & $\begin{array}{c}\text { IV. } \\
\text { Bahn }\end{array}$ & $\begin{array}{c}\text { Stromdichte } \\
\text { im 4. Bahnmax. } \\
\text { bei 300 } \mu \mathrm{A} \\
H F \text {-Strom } \\
\text { in } \mu \mathrm{A} / \mathrm{mm}^{2} \text { (75 V) }\end{array}$ \\
\hline $0^{\circ}$ & 100 & 73 & 45 & 40 & 0,8 \\
$45^{\circ}$ & 100 & 95 & 82 & 86 & 2,1 \\
$90^{\circ}$ & 100 & 87 & 79 & 59 & 1,1 \\
$135^{\circ}$ & $10)$ & 71 & 53 & 29 & 0,5 \\
$180^{\circ}$ & 100 & 50 & 32 & 22 & 0,5 \\
\hline
\end{tabular}

Tab. 1. Relative Anzahl der Elektronen, die in den Bahnen I-IV kreisen, bei verschiedenen Einfallswinkeln $\vartheta$ (75 V HF-Spannung); maximale Stromdichte in $\mu \mathrm{A} / \mathrm{mm}^{2}$ in der IV. Bahn bei $300 \mu \mathrm{A} \mathrm{HF-Strom.}$

Hier sind die Elektronenströme, die in den Bahnen I bis IV kreisen, in Abhängigkeit von $\vartheta$ angegeben. Dabei ist jeweils die Zahl der Elektronen, die in der ersten Bahn laufen, gleich 100 gesetzt, um von Änderungen in der Kathodenemission zwischen den einzelnen Versuchsreihen frei zu werden. Für $\vartheta=0^{\circ}$ findet die eine Hälfte der Elektronen beim Spaltdurchtritt eine Sammellinse, die andere ein Gegenfeld vor. Der Intensitätsverlust beträgt demgemäß von der I. bis zur IV. Bahn $60 \%$. Für $\vartheta=45^{\circ}$ treffen alle Elektronen auf eine Sammellinse, der Intensitätsverlust bleibt deshalb sehr klein. Bei $\vartheta=90^{\circ}$ treffen die Elektronen zur Hälfte auf eine Sammellinse und zur Hälfte auf eine Zerstreuungslinse. Es wird demnach, wie schon im Abschn. 2 festgestellt wurde, etwa die Hälfte der Elektronen aus der Bahn ausgeschieden. Für $\vartheta=135^{\circ}$ finden alle Elektronen eine Zerstreuungslinse beim Spaltdurchtritt, während bei $\vartheta=180^{\circ}$ die eine Hälfte der Elektronen auf eine Zerstreuungslinse, die andere auf ein Gegenfeld trifft. In beiden Fällen gehen bis zur 4. Bahn schon etwa 3/4 der am Anfang kreisenden Elektronen verloren. Die letzte Spalte der Tab. 1 enthält die maximale Stromdichte, die in der letzten
Bahn auftritt, wenn der $H F$-Strom auf die Halbdosen $300 \mu \mathrm{A}$ beträgt. Auch hieraus läßt sich die starke Überlegenheit der Winkelstellung $\vartheta=45^{\circ}$ bezüglich der Fokussierungs-Eigenschaften ablesen. Die theoretischen Erwartungen werden also durch das Experiment vollauf bestätigt. Es lassen sich im Elektronenzyklotron bei richtiger Wahl des Eintrittswinkels der Elektronen in den Halbdosenraum annähernd alle Elektronen, die überhaupt aus der Kathode herausgesaugt werden können, durch den Bereich des Magnetfeldes bringen, in dem die elektrische Fokussierung allein maßgebend ist.

Zum Schluß sollen noch die Elektronen betrachtet werden, deren Einfallsrichtungen in einer senkrechten Ebene zu der bisher behandelten Mittelebene der Halbdosen liegen und mit der Horizontalen den Winkel $\varphi$ einschließen, während $\vartheta$ einem Winkel von $90^{\circ}$ entsprechen möge. Qualitativ gewinnt man leicht einen Überblick, wenn man die Aufnahmen VII und VIII der Abb. 2 betrachtet. Bild VII zeigt die Seitenansicht der Elektronenbahnen, wenn das übliche Kathodensystem verwandt wird. Die Elektronen verlassen die Kathode in der senkrecht stehenden Ebene unter allen möglichen Winkeln. Sie sind ober- und unterhalb der Mittelebene symmetrisch verteilt und reichen in den Bahnmaxima von der unteren bis zur oberen Halbdosenfläche. Bild VIII zeigt die gleiche Ansicht wie VII, nur ist hier der Elektronenstrahl scharf gebündelt und tritt unter einem Winkel von $\vartheta=16^{\circ}$ gegen die Mittelebene in die Halbdosen ein. Der Elektronenstrahl verläuft zunächst, von der Kathode ausgehend, nach rechts oben und nach dem ersten Spaltdurchtritt weiter nach links oben. Hier wird ein kleiner Bruchteil der Elektronen aus dem Halbdosenraum abgelenkt. Die Masse der Elektronen wird aber durch das an der Halbdosenkante besonders stark brechende $\mathrm{HF}$ Feld nach unten zurückgebogen. Die folgenden Umläufe richten sich weiter nach unten. Ein derartiges Pendeln der außerhalb der Mittelebene laufenden Bahnen hat Wils o ${ }^{3}$ beschrieben. Danach muß man erwarten, daß die Elektronen sich bei weiteren Umläufen wieder nach oben wenden und so fort. Man sieht daraus, daß noch unter beträchtlichen Winkeln in der senkrechten Ebene die Elektronen innerhalb der Halbdosen beschleunigt werden können. Es empfiehlt sich daher, den Halbdosen auch schon in der Mitte des Magnetfeldes eine große innere Weite zu geben. 
Zusammenfassend läßt sich sagen, daß im vorliegenden Fall ein Raumwinkel von $30^{\circ}$ in der Höhe und $30^{\circ}$ in der Breite für den Elektroneneinfall nutzbar gemacht werden kann. Denn auch der Winkel $\vartheta$ in der Bahnebene läßt einen so breiten Spielraum zu, ohne die Zahl der fokussierbaren Elektronen wesentlich $\mathrm{zu}$ vermindern. In einem derartig großen Raumwinkel können aber praktisch alle Teilchen einer Quelle fokussiert werden.
Die hier am Elektronenzyklotron gesammelten Erfahrungen lassen sich natürlich auf ein Zyklotron, das mit Ionen arbeitet, übertragen. Der Übergang zu schweren Ladungsträgern bedingt aber die Anwendung von Ionenquellen, deren Aufbau von einer Elektronenquelle in Geometrie und Absaugbedingung abweicht, so daß für eine solche Übertragung der Ergebnisse eine sorgfältige Prüfung der veränderten Bedingungen notwendig wird.

\title{
Ein 300-kV-Bandgenerator mit übernormaler Ladungsdichte
}

\author{
Von Erwin Willy Becker \\ Aus dem Kaiser-Wilhelm-Institut für Chemie, Tailfingen \\ (Z. Naturforschg. 2a, 395-397 [1947]; eingegangen am 1. Mai 1947)
}

Es wird ein $300 \mathrm{kV}$ van-de-Graaff-Generator Kossel-Heise'scher Bauart beschrieben, der in Luft von Atmosphärendruck mit einer Beladungsdichte von 18 el.st. cgs-Einheiten $/ \mathrm{cm}^{2}$ arbeitet.

$\mathrm{E}$ lektrostatische Bandgeneratoren sind seit ihrer ersten Beschreibung durch van de Graaff und Mitarb. ${ }^{1}$ hauptsächlich für kernphysikalische Untersuchungen verwendet worden. Es wurden dabei Spannungen von mehreren Millionen Volt und Stromstärken von einigen Milliampère erreicht. Außer bei diesen großen Apparaten, die naturgemäß nur mit erheblichem Aufwand gebaut und betrieben werden können, läßt sich das van-de-Graaff-Prinzip auch bei wesentlich kleineren Maschinen verwenden, die dann für Laboratoriumszwecke häufig sehr brauchbare und billige Hochspannungsquellen darstellen. Der Aufwand zur Erzeugung von einigen hundert $\mathrm{kV}$ wird besonders gering, wenn die Bandbesprühung durch Selbsterregung erfolgt, eine Möglichkeit, die insbesondere von W. Kossel und seinen Mitarbeitern ${ }^{2}$ ausgenutzt wurde.

Die von elektrostatischen Generatoren gelieferte Stromstärke ist verhältnismäßig klein. Da das freie Band in Luft von Atmosphärendruck maximal mit 8 el.st. cgs-Einheiten pro $\mathrm{cm}^{2}$ beladen werden kann ${ }^{3}$ und in der Praxis nur 50 bis $60 \%$ da-

1 R. J. van de Graaff, K. T. Compton u. L. C. van Atta, Physic. Rev. 43, 149 [1933]; Literatur bis 1939 s. W. Gentner, Die Erzeugung schneller Ionenstrahlen für Kernreaktionen, Ergebn. exakt. Naturwiss. 19, 107 [1940].

2 W. Kossel, Z. Physik 111, 264 [1938]; U. Ne u bert, Z. Physik 110, 334 [1938]. von $\mathrm{zu}$ erreichen sind, ist zur Erzeugung von $100 \mu \mathrm{A}$ bereits eine Flächengeschwindigkeit von etwa $3 \mathrm{~m}^{2} / \mathrm{sec}$ erforderlich. Die Anwendung von Druckluft, die zu höheren Beladungsdichten führt, ist bei kleinen Mașchinen wegen des unverhältnismäßig großen Aufwandes nicht wirtschaftlich. Es ist daher von Interesse, daß sich nach Kossel und $\mathrm{Heise}^{4}$ in Luft von Atmosphärendruck die 8 cgs nicht nur tatsächlich erreichen, sondern sogar überschreiten lassen, wenn man die beiden Bandhälften aufeinander gleiten läßt. Kossel und Heise erzielten so Beladungsdichten von $12 \mathrm{cgs}$.

Ein ähnlicher, mit ,übernormaler“ Ladungsdichte arbeitender $300-\mathrm{kV}$-Generator, der sich bei gaskinetischen Untersuchungen ${ }^{5}$ in mehrjährigem Betrieb gut bewährt hat, sei im folgenden kurz beschrieben. Durch Einführung eines weiteren Spitzenkammes konnte die von $\mathrm{Kossel}$ und Heise erreichte Beladungsdichte überschritten und mit $18 \mathrm{cgs}$ die nach unserem Wissen bisher höchste Flächendichte in Luft von Atmosphärendruck erreicht werden.

3 W. Kossel ${ }^{2}$, S. 272.

4 W. Kossel u. F. Heise, Z. Physik 113, 769 [1939]; F. He is e, Z. Physik 116, 317 [1940].

5 E. W. B e c k e r , Z. Naturforschg. 2a, 297 [1947]; ebenda „Das Trennrohr mit hohem Druck und radialem elektrischen Feld“, erscheint demnächst. 